\title{
Experimental investigation on vibration and acoustic similarity of stiffened cylindrical shells
}

\author{
Bin Fang ${ }^{1,}$ a , Hanqin $\mathrm{Li}^{2, b}$ and Zhemin $\mathrm{Jin}^{3, \mathrm{c}}$ \\ ${ }^{1,2,3}$ College of Warship and Ocean, Naval University of Engineering, No.717 Jiefang Road, Wuhan, \\ 430033, China \\ arobin_fb@163.com, b58479221@qq.com, c763487861@qq.com
}

\begin{abstract}
Keywords: vibration, acoustics, similarity, siffened cylindrical shells.
Abstract. The employment of similitude theory to establish similarity among structural-acoustic systems can save considerable expense and time, provided that the proper scaling laws are found and validated. In this study the scaling laws for structural-acoustic systems are developed and validated. The stiffened cylindrical shells of the model and prototype are fabricated, and their frequency response functions (FRFs) of acceleration and sound pressure subjected to a random excitation are measured in a semi-anechoic chamber. The difficulty of making completely similar structures often leads to certain types of relaxations and distortions between the model and prototype. In order to overcome this problem, the equivalent thickness of stiffened shells and the non-dimensional quantities, including non-dimensional frequency and non-dimensional FRFs are introduced. The obtained results show that the FRFs of the model and prototype closely resemble each other compared throughout the frequency range and the scaling laws provide precise relationship between a full-scale prototype and its small-scale equivalent model.
\end{abstract}

\section{Introduction}

Dynamic response characteristics of structural-acoustic systems are one of the fundamental requirements in designing and evaluating noise control measures. Finding dynamic response, including frequency response functions (FRFs) of acceleration and sound pressure, through performing acoustic test in case of heavy and huge systems, called the prototype, is very difficult to do and also requires advanced and huge test instrumentations and considerable expense and time. The employment of similitude theory to establish similarity among structural-acoustic systems can save considerable expense and time, if the proper scaling laws are found and validated. Similarity conditions or scaling laws provide the relationship between the prototype and its scale models. This relationship must be based on the existence of certain parameters that control the behavior of the prototype. If such parameters exist, a scaled down model can be built, which will duplicate the response of the full scale prototype. The two systems are then said to be similar. The theory of similitude includes a consideration of the conditions under which the behavior of two separate systems will be similar, and the techniques of accurately predicting results on the one from the observations to the other.

Considering the significant role of stiffened cylindrical shell in various types of structural-acoustic systems, practical application of scaled down stiffened cylindrical shells and the importance of establishing a similarity and finding proper scaling laws will be evident. Due to the large number of design parameters in stiffened cylindrical shells, the identification of the principal scaling laws only through dimensional analysis is difficult. Similitude theory based on the governing equation of structural-acoustic systems is more direct and simpler in execution, so both the dimensional analysis and the equations analysis will be used in this paper.

Some studies particularly concerning the use of scaled down shell structures have been conducted in the past. Soedel investigated similitude requirements for vibrating thin shells [1]. Prediction of shell vibration response in cross-ply cylindrical shells using scaled down models, based on partial similitude was accomplished by Rezaeepazhand [2]. In a similar investigation presented by the same authors, vibration response of laminated cylindrical shells with double curvature was investigated 
based on structural similitude [3]. Effect of extensive use of welding on buckling behavior of large cylindrical shells constructed from a large number of curved panels was investigated experimentally using scale models by Teng [4]. Wang used dimensional theory to obtain the similarity conditions between model and prototype for an underwater stiffened cylinder [5]. Jha developed the necessary similarity conditions for dynamic testing of scaled structures, and these scaling laws ware validated by carrying out the finite element analysis and experimental studies on simple structures [6]. Torkamani used the Donnell-type nonlinear strain-displacement relations along with the smearing theory to develop the similitude theory for free vibrations of orthogonally stiffened cylindrical shells [7]. And in order to overcome the difficulties encountered in fabricating small-scaled stiffeners, design of equivalent cross section was explored that in spite of having much simpler profile, offers the same vibration effect in free vibration of stiffened shells. A scaling approach to predict high-frequency mean responses of vibration systems was analyzed by Li [8]. Many research activities had been conducted on scale-down modeling of dynamic and static behavior of other structural systems. Rosa proposed a similitude for analysis of the dynamic response of acousto-elastic assemblies, invoking the energy distribution approach which allows the exact representation of all the fundamental parameters in terms of modal coordinates [9]. The scaling laws for physical modeling of anti-symmetric cross-ply laminated circular cylindrical shells for buckling and free vibration were developed by Variddhi, and several possible model of partial similitude are investigated numerically $[10,11]$. Wu presented a technique for predicting the vibration characteristics of an elastically supported full-size flat plated subjected to circular-moving loads from those of its complete-similitude scale model and the associated scaling laws [12].

In the present study, scaling laws have been derived for the dynamic analysis of structural-acoustic system and different types of possible relaxations are taken into consideration to meet the scaling conflicts encountered for dynamic analysis. For the purpose of experimental validation of the methodology, stiffened cylindrical shells of the model and prototype are fabricated, and their FRFs of acceleration and sound pressure subjected to a random excitation are measured in a semi-anechoic chamber. The difficulty of making completely similar structures often leads to certain types of relaxations and distortions between the model and the prototype. In order to overcome this problem, the equivalent thickness of stiffened shells and the non-dimensional quantities, including non-dimensional frequency and non-dimensional FRFs are introduced. The obtained results show that the FRFs of the model and the prototype closely resemble each other compared throughout the frequency range.

\section{Scaling laws of stiffened cylindrical shells}

Scaling laws can be confined to the following steps: list all relevant variables involved in the physical problem; establish by dimensional analysis all pi-terms; list the given values and (or) ranges of prototype variables; and establish the scaling factors for all variables. For the forced vibration of damped multiple-degrees-of-freedoms systems, governing equation can be expressed as Eq. 1:

$$
[M]\{+[C]\{l \&+[K]\{u\}=\{F\} .
$$

where $M$ is the mass matrix of the structure, $C$ is the damping coefficient matrix, $K$ is the stiffness matrix, $\{u\}$ is the column matrix for displacement, $\{u\}$ is the first-order derivative for displacement, $\{$ is the second-order derivative for displacement, $\{F\}$ is the column matrix of applied force including the excitation force.

The governing equation for acoustic radiation of structure can be expressed as Eq. 2:

$$
\nabla^{2} p-\frac{1}{c_{0}^{2}} \frac{D^{2}}{D t^{2}} p=\nabla \cdot \boldsymbol{F}-\rho_{0} \frac{D q}{D t} .
$$


where $p$ is the pressure, $\rho_{0}$ is the density of the fluid, $c_{0}$ is the speed of sound, $\boldsymbol{F}$ denotes an externally applied volume force, $q$ idenotes an external volume flow source within the fluid.

Stiffened cylindrical shell must be designed, loaded and interpreted according to the scaling laws that relate the model to the prototype. Replica model is a physical model of the prototype which is geometrically similar in all aspects to the prototype and employs identically the same materials at similar locations. The BCs (boundary conditions) and loading conditions are similar for model and prototype. In the case of similarity conditions being satisfied, the non-dimensional frequency parameter defined as below [7]

$$
\Omega=\omega \sqrt{\frac{\bar{M} l_{c}^{4}}{D}} .
$$

will have the same value in model and prototype, where $\bar{M}$ is the averaged mass per unit area of the stiffened cylindrical shell, $l_{c}$ is the length of cylinder.

The non-dimensional acceleration $\Lambda$ and non-dimensional radiation sound pressure $\Theta$ defined as below

$$
\Lambda=A M / F_{0}, \Theta=p L_{d}^{2} / F_{0} .
$$

will have the same value in model and prototype when the complete similarity is achieved, where $L_{d}$ is the distance between microphones and the center of cylinder and $M$ is the mass of stiffened cylindrical shell.

\section{Experimental validation of the scaling laws}

For the purpose of experimental validation of the methodology as applied here, two cylindrical shells are fabricated. The first shell denoted as prototype is a stiffened cylindrical shell made of steel ( $E=206 \mathrm{Gpa}, \rho=7850 \mathrm{~kg} / \mathrm{m}^{3}, v=0.3$ ), having nine equally spaced internal rectangle shape ribs (Fig.1). The second shell denoted as model is a 1/2-scale model of the prototype, made of steel, which is the scale down of the prototype on geometry. The geometrical parameters of the prototype and model are showed in Table 1.

\begin{tabular}{|c|c|c|c|c|c|c|}
\hline \multirow{2}{*}{ Parameter } & \multirow{2}{*}{ Symbol } & \multirow{2}{*}{ Unit } & \multicolumn{2}{|c|}{ Model } & \multicolumn{2}{|c|}{ Prototype } \\
\hline & & & design & actual & design & actual \\
\hline radius of cylinder & $R$ & $\mathrm{~mm}$ & 403 & 401.2 & 806 & 805 \\
\hline surface area of cylinder & $A$ & $\mathrm{~m}^{2}$ & 3.60 & 3.58 & 14.41 & 14.38 \\
\hline length of cylinder & $l_{c}$ & $\mathrm{~mm}$ & 1020 & 1018 & 2040 & 2039.3 \\
\hline thickness of shell & $t_{s}$ & $\mathrm{~mm}$ & 3 & 2.58 & 6 & 5.69 \\
\hline thickness of cover or bulkhead & $t_{c}$ & $\mathrm{~mm}$ & 10 & 9.48 & 20 & 19.52 \\
\hline thickness of rib & $t_{r}$ & $\mathrm{~mm}$ & 5 & 4.82 & 10 & 9.94 \\
\hline rib spacing & $l_{r}$ & $\mathrm{~mm}$ & 100 & 100 & 200 & 200 \\
\hline rib height & $h_{r}$ & $\mathrm{~mm}$ & 25 & 23.95 & 50 & 51.16 \\
\hline radius of the hatch on cylinder & $R_{h}$ & $\mathrm{~mm}$ & 225 & 225 & 450 & 450 \\
\hline radius of cover & $R_{c}$ & $\mathrm{~mm}$ & 315 & 315.5 & 630 & 628.5 \\
\hline $\begin{array}{c}\text { equivalent thickness of stiffened } \\
\text { cylindrical shell }\end{array}$ & $t_{e}$ & $\mathrm{~mm}$ & 9.389 & 8.834 & 18.778 & 19.088 \\
\hline mass & $M$ & $\mathrm{~kg}$ & 178 & 169 & 1427 & 1387 \\
\hline $\begin{array}{c}\text { averaged mass per unit area of } \\
\text { cylinder }\end{array}$ & $\bar{M}$ & $\mathrm{~kg} / \mathrm{m}^{2}$ & 49.53 & 47.27 & 99.06 & 96.46 \\
\hline
\end{tabular}

Table 1. Parameters of the prototype and model of the stiffened cylindrical shells 

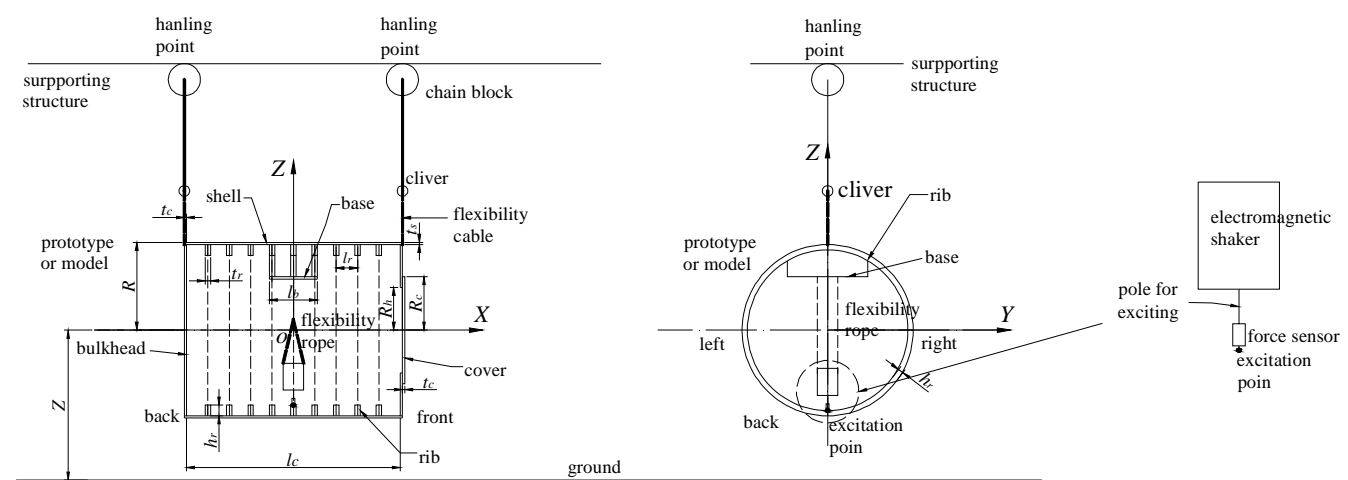

Fig. 1 Geometry of the stiffened cylindrical shell for experimental validation

For difficulties in fabricating of precise small-scaled stiffened cylindrical shell, deviation between design structure and actual structure cannot be avoided. The actual values of fabricated stiffened cylindrical shells have been measured and listed in Table 1. Comparing between the design values and actual values, the deviations are less than ten percent, except the shell thickness. Method must be found to overcome the problem of the fabrication imperfection on similitude between the model and prototype. The equivalent thickness will be introduced for non-dimension frequency in Eq. 3. The shell with ribs will be translated to the shell without ribs by equivalent thickness ("shell-e" in Eq. 4), which has the same cross-section moment of inertia. The equivalent thickness of model and prototype can be computed by Eq. 4.

$$
\left(I_{x}\right)_{\text {shell }}+\left(I_{x}\right)_{\text {ribs }}=\left(I_{x}\right)_{\text {shell-e }} \Rightarrow \frac{1}{12} l_{c} t_{s}^{3}+9\left(\frac{1}{12} t_{r} h_{r}^{3}+t_{r} h_{r} \cdot\left(\frac{1}{2} h_{r}+\frac{1}{2} t_{s}\right)^{2}\right)=\frac{1}{12} l_{c} t_{e}{ }^{3} \text {. }
$$

A two cable soft suspension system is used to provide free-free boundary condition for the model and the prototype, which is showed in Fig. 2. An electromagnetic exciter driven by a power amplifier, and generating a random excitation, is used to exciting the model and the prototype into vibration (Fig. 1). The electromagnetic exciter is hanged in the cylinder shell by four soft ropes. The exciters for the model and prototype are different. The small exciter, who's mass is $3.5 \mathrm{~kg}$ and maximum excitation force is $50 \mathrm{~N}$, is used to exciting the model. The big exciter, who's mass is $26 \mathrm{~kg}$ and maximum excitation force is $500 \mathrm{~N}$, is used to exciting the prototype.
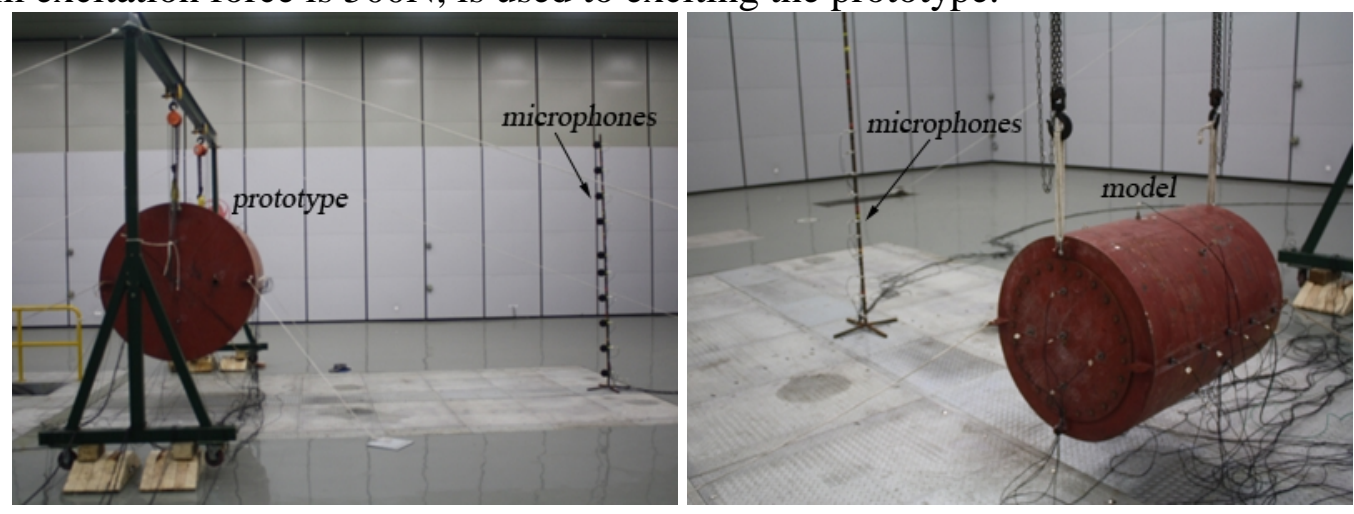

Fig. 2 Prototype and model of the stiffened cylindrical

The excitation force is measured by a force transducer. The acceleration response is measured using piezoelectric accelerometers in the longitudinal and radial directions on 38 points of the prototype and the corresponding points of the model. The sound pressure is measured by microphones on lateral directions on 10 points of the prototype and the corresponding points of the model. All the signals are collected by the signal collection devices and analyzer software is used to extract FRFs. The location of measurement points of the prototype and model are shown in Fig. 3. 


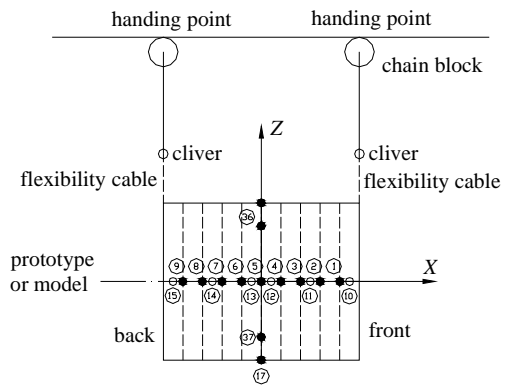

ground

side view

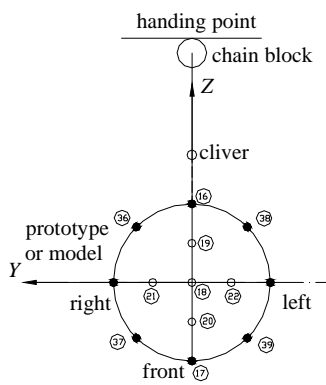

front view

Fig. 3 Acceleration and sound pressure measurement points of the test on the prototype or model

As seen before, in the case of complete similarity conditions being satisfied, the non-dimensional frequency, the non-dimensional FRF of acceleration and sound pressure will have the same value in model and prototype. Thus, if the FRFs of model and prototype are plotted versus non-dimensional frequency, both of the FRFs should yield similar behavior. Therefore, the vibration response and acoustic radiation of the model and the prototype can be compared throughout the frequency range of excitation. A number of such plots, comparing the FRFs of some distinct points on the prototype and their corresponding points on the model, measured during the test are presented in Fig. 4 and Fig. 5. The actual values should be used in the non-dimension frequency (Eq. 3). Therefore, the vibration response of the actual model and the actual prototype can be compared throughout the frequency range of excitation.
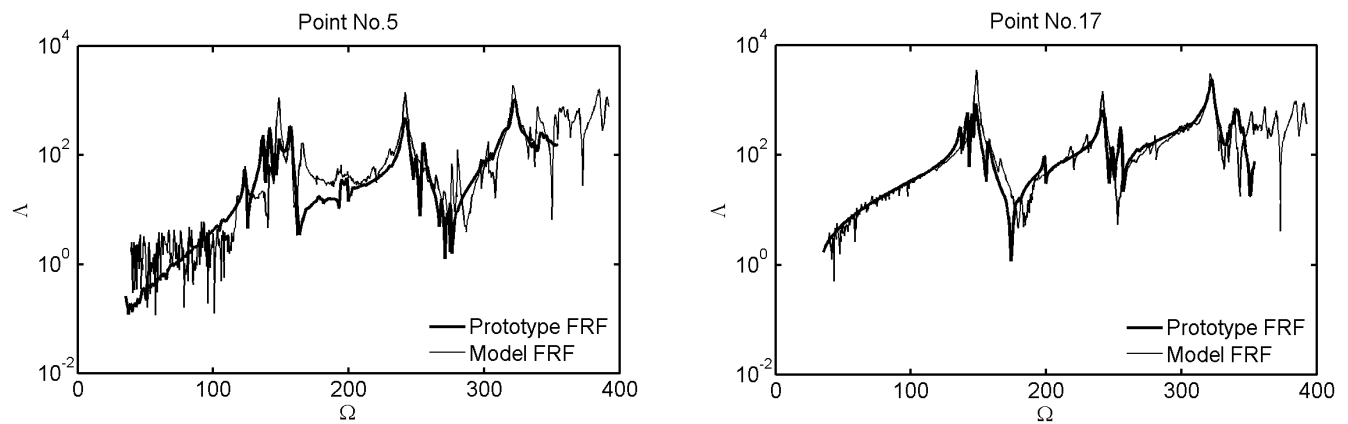

Fig. 4 Acceleration FRFs of prototype and model.
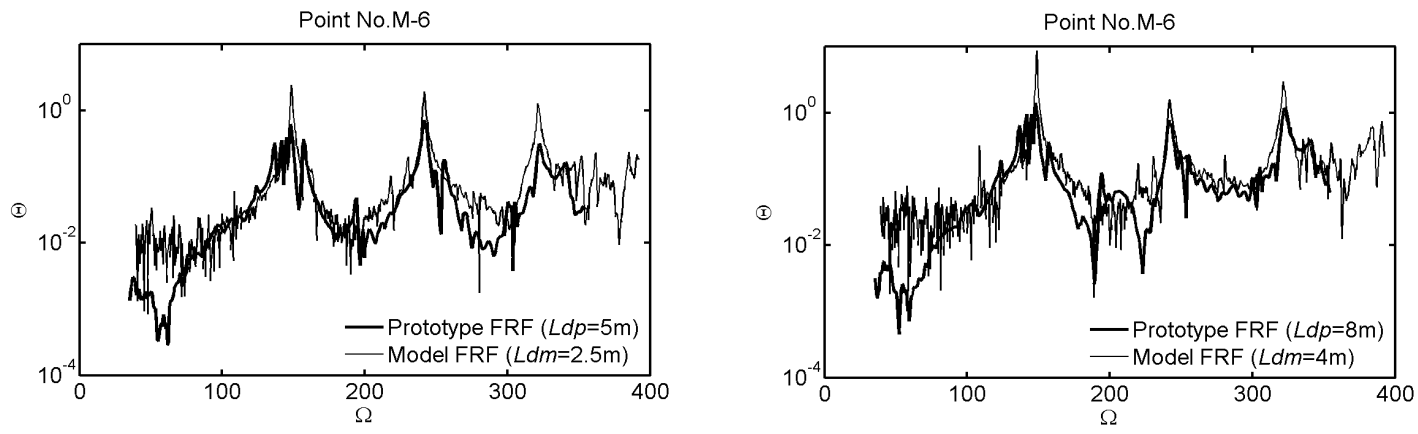

Fig. 5 Sound pressure FRFs of prototype and model.

The behavior of the actual model and the actual prototype closely resemble each other, but in some cases such as the response amplitude peaks there exists inconsistency. In this case, since the less-than-perfect value is occurred for the response amplitude peaks, the reason probably is the dissimilar of damping coefficient between the model and the prototype.

\section{Conclusions}

In this study, the necessary similarity conditions or scaling laws for dynamic analysis of stiffened cylindrical shell are developed. Based on the similarity conditions or scaling laws, the relation between the acceleration and the sound pressure of a scale model and those of its prototype are 
obtained. For the purpose of experimental validation of the methodology, stiffened cylindrical shells of the model and prototype are fabricated, and their FRFs of acceleration and sound pressure subjected to a random excitation are measured in a semi-anechoic chamber. The difficulty of making completely similar structures often leads to certain types of relaxations and distortions between the model and the prototype. In order to overcome this problem, the equivalent thickness of stiffened shells and the non-dimensional quantities, including non-dimensional frequency and non-dimensional FRFs are introduced. The obtained results show that the FRFs of the model and the prototype closely resemble each other compared throughout the frequency range. It means that the scaling laws provide precise relationship between a full-scale prototype and its small-scale equivalent model for the frequency characteristics of dynamic response, and can be used to extrapolate the experimental data of a small inexpensive and testable model into design information for a large prototype.

\section{Acknowledgment}

Authors would like to gratefully acknowledge Mr. Bo Wang, Mr. Qiongfeng Xu, Mr. Chao Li and Mr. Lu Tan for their effort in doing experiments.

\section{References}

[1] W. Soedel: Similitude Approximations for Vibrating Thin Shells. J. Acoust. Soc. Am. Vol. 49(1971), p.1535-1541.

[2] J. Rezaeepazhand, G.J. Simitses, J.H. Starnes: Design of scaled down models for predicting shell vibrations response. J. Sound Vib. Vol. 195 (1996), p. 301-311.

[3] J. Rezaeepazhand, G.J. Simitses: Structural similitude for vibration response of laminated cylindrical shells with double curvature. Composites Part B Vol. 28(1997), p. 195-200.

[4] J. G. Teng, X Lin: Fabrication of small models of large cylinders with extensive welding for buckling experiments. Thin Wall Struct. Vol. 43(2005), p. 1091-114.

[5] S. Wang: Study on the acoustic similitude of the underwater complex shell structure. J. Acoust. Soc. Am. Vol. 119(2006), p. 3419-3419.

[6] A. Jha, R. Sedaghati, R. Bhat: Analysis and dynamic testing of structures subjected to vibration and shock using scale models. Canadian Aeronautics and Space Journal. Vol. 52(2006), p. 95-108.

[7] Sh. Torkamani, H.M. Navazi, A.A. Fafari, M. Bagheri: Structural similitude in free vibration of orthogonally stiffened cylindrical shells. Thin Wall Struct. Vol. 47(2009), p. 1316-1330.

[8] Xianhui Li: A scaling approach for the prediction of high-frequency mean responses of vibration systems. J. Acoust. Soc. Am. Vol. 127(2010), p. 209- 214.

[9] S.D. Rosa, F. Franco, X. Li, T. Polito: A similitude for structural acoustic enclosures. Mech. Syst. Signal Pr. Vol. 30 (2012), p. 330-342.

[10]U. Variddhi, S. Pairod: Similitude invariants and scaling laws for buckling experiments on anti-symmetrically laminated plated subjected to biaxial loading. Compos. Struct. Vol. 59(2003), p. $455-465$.

[11]U. Variddhi, W. Nuttawit: Structural similitude and scaling laws of anti-symmetric cross-ply laminated cylindrical shells for bucking and vibration experiments. Int. J. Str. Stab. Dyn. Vol. 7(2007), p. 609-627..

[12] J.J. Wu: Prediction of the dynamic characteristics of an elastically supported full-size flat plate from those of its complete-similitude scale model. Comput. Struct. Vol. 84(2006), p. 102-114. 